\title{
The volume flux group and nonpositive curvature
}

\author{
Pablo Suárez-Serrato
}

Received: 1 August 2008 / Accepted: 13 November 2008 / Published online: 3 December 2008 (C) Springer Science+Business Media B.V. 2008

\begin{abstract}
We show that every closed nonpositively curved manifold with non-trivial volume flux group has zero minimal volume, and admits a finite covering with circle actions whose orbits are homologically essential. This proves a conjecture of Kedra-Kotschick-Morita for this class of manifolds.
\end{abstract}

Keywords Volume flux group $\cdot$ Minimal volume $\cdot$ Nonpositive curvature

Mathematics Subject Classification (2000) Primary 57R50 - 57S05 · 53C23 - Secondary $53 \mathrm{C} 21 \cdot 22 \mathrm{E} 65$

Let $M$ be a closed smooth manifold and $\mu$ a volume form on $M$. Denote by Diff ${ }^{\mu}$ the group of $\mu$-preserving diffeomorphisms of $M$, and by Diff ${ }_{0}^{\mu}$ its identity component. The $\mu$-flux homomorphism Flux $_{\mu}$, from the universal covering $\widetilde{\operatorname{Diff}_{0}^{\mu}}$ to the $(n-1)$-cohomology group $H^{n-1}(M ; \mathbf{R})$, is defined by the formula

$$
\operatorname{Flux}_{\mu}\left(\left[\varphi_{t}\right]\right)=\int_{0}^{1}\left[i_{\dot{\varphi}_{t}} \mu\right] \mathrm{d} t .
$$

It induces a homomorphism

$$
\operatorname{Flux}_{\mu}: \pi_{1}\left(\operatorname{Diff}_{0}^{\mu}\right) \rightarrow H^{n-1}(M ; \mathbf{R})
$$

whose image is the volume flux group $\Gamma_{\mu} \subset H^{n-1}(M ; \mathbf{R})$. The $\mu$-flux homomorphism descends to a homomorphism

$$
\operatorname{Flux}_{\mu}: \operatorname{Diff}_{0}^{\mu} \rightarrow H^{n-1}(M ; \mathbf{R}) / \Gamma_{\mu} .
$$

P. Suárez-Serrato

Mathematisches Institut LMU, Theresienstrasse 39, München 80333, Germany

P. Suárez-Serrato $(\varangle)$

CIMAT, CP 36240, Guanjuato, GTO, México

e-mail: p.suarez-serrato@cantab.net 
For a closed connected smooth Riemannian manifold $(M, g)$, let $\operatorname{Vol}(M, g)$ denote the volume of $g$, and let $K_{g}$ be its sectional curvature. We define the minimal volume of $M$ following Cheeger and Gromov [2]:

$$
\operatorname{Min} \operatorname{Vol}(M):=\inf _{g}\left\{\operatorname{Vol}(M, g):\left|K_{g}\right| \leq 1\right\}
$$

The minimal volume is a very sensitive invariant; it was first observed by Bessières [1] that its value may depend on the differentiable structure of $(M, g)$. Indeed, Kotschick [6] has shown that even the vanishing of $\operatorname{Min} \operatorname{Vol}(M)$ can detect changes in the smooth structure of M.

The investigation of relationships between the volume flux group of $M$ and various invariants which bound $\operatorname{Min} \operatorname{Vol}(M)$ from below was set in motion by Kedra et al.[5]. They put forward the idea that if a closed manifold has non-trivial volume flux group, then its minimal volume should vanish. The aim of this report is to verify that statemtent for closed manifolds which carry a metric of nonpositive sectional curvature.

The volume flux group $\Gamma_{\mu}$ is independent of the form $\mu$-see Sect. 3 of [5] - so it can be considered as an invariant of the manifold $M$ itself.

Theorem 1 Every closed nonpositively curved manifold with non-trivial volume flux group has zero minimal volume.

The technique we will use to show that the minimal volume vanishes is an $\mathcal{F}$-structure, which was introduced by Gromov as a generalisation of an $S^{1}$-action.

Definition 2 An $\mathcal{F}$-structure on a closed manifold $M$ is given by the following conditions.

(1) A finite open cover $\left\{U_{1}, \ldots, U_{N}\right\}$

(2) $\pi_{i}: \widetilde{U}_{i} \rightarrow U_{i}$ a finite Galois covering with group of deck transformations $\Gamma_{i}, 1 \leq i \leq N$

(3) A smooth torus action with finite kernel of the $k_{i}$-dimensional torus,

$$
\phi_{i}: T^{k_{i}} \rightarrow \operatorname{Diff}\left(\widetilde{U}_{i}\right), 1 \leq i \leq N
$$

(4) A homomorphism $\Psi_{i}: \Gamma_{i} \rightarrow \operatorname{Aut}\left(T^{k_{i}}\right)$ such that

$$
\gamma\left(\phi_{i}(t)(x)\right)=\phi_{i}\left(\Psi_{i}(\gamma)(t)\right)(\gamma x)
$$

for all $\gamma \in \Gamma_{i}, t \in T^{k_{i}}$ and $x \in \widetilde{U}_{i}$

(5) For any finite sub-collection $\left\{U_{i_{1}}, \ldots, U_{i_{l}}\right\}$ such that $U_{i_{1} \ldots i_{l}}:=U_{i_{1}} \cap \ldots \cap U_{i_{l}} \neq \emptyset$ the following compatibility condition holds: let $\widetilde{U}_{i_{1} \ldots i_{l}}$ be the set of points $\left(x_{i_{1}}, \ldots, x_{i_{l}}\right) \in \widetilde{U}_{i_{1}}$ $\times \cdots \times \widetilde{U}_{i_{l}}$ such that $\pi_{i_{1}}\left(x_{i_{1}}\right)=\cdots=\pi_{i_{l}}\left(x_{i_{l}}\right)$. The set $\widetilde{U}_{i_{1} \ldots i_{l}}$ covers $\pi_{i_{j}}^{-1}\left(U_{i_{1} \ldots i_{l}}\right) \subset \widetilde{U}_{i_{j}}$ for all $1 \leq j \leq l$, and we require that $\phi_{i_{j}}$ leaves $\pi_{i_{j}}^{-1}\left(U_{i_{1} \ldots i_{l}}\right)$ invariant and it lifts to an action on $\widetilde{U}_{i_{1} \ldots i_{l}}$ such that all lifted actions commute

An $\mathcal{F}$-structure is said to be pure if all the orbits of all actions at a point, for every point have the same dimension. We will say an $\mathcal{F}$-structure is polarised if the smooth torus action $\phi_{i}$ above is fixed point free for every $U_{i}$. The existence of a polarised $\mathcal{F}$-structure on a manifold $M$ implies the minimal volume $\operatorname{MinVol}(M)$ is zero by the main result of Cheeger and Gromov [2]. The interested reader is invited to consult the illuminating examples found there as well.

The attentive reader will notice that the definition of an $\mathcal{F}$-structure above is different from the sophisticated one found in [2]. Despite this, it is sufficiently practical to be implemented 
and also satisfies the properties needed in the proof that a polarised $\mathcal{F}$-structure forces the minimal volume to vanish, which can be consulted in [2].

Proof of Theorem 1. Let $M$ be a compact nonpositively curved manifold whose volume flux group is not trivial. The fundamental group $\pi_{1}(M)$ has non-trivial centre $Z$, a proof of which can be found in [5, Theorem 15] and compared with work of Fathi [4, Proposition 5.1] and the various references and attributions contained therein. In this case $M$ admits a finite covering space $M^{*}$ diffeomorphic to $T^{k} \times N$, where $T^{k}$ is a flat torus of dimension $k$ and $N$ is a compact nonpositively curved manifold as was shown by Eberlein [3].

Even though it may seem that this already implies $\operatorname{Min} \operatorname{Vol}(M)=0$, for completeness will now show how to construct a pure polarised $\mathcal{F}$-structure on $M$; since the torus $T^{k}$ splits off $M^{*}$ smoothly and the action of $T^{k}$ on itself as a factor of $M^{*}$ is compatible with the covering transfomation in the required sense. This will also provide an example of a detailed construction of a pure polarised $\mathcal{F}$-structure.

Represent $M$ as $H / \Gamma$, where $H$ is simply connected and $\Gamma$ is a properly discontinuous group of isometries of $H$ which acts freely. The space $H$ decomposes into $H_{1} \times H_{2}$, where $H_{1}=\mathbf{R}^{k}$ is a Euclidean space of dimension $k=\operatorname{rank}(Z)$.

Notice that every element $\gamma$ of $\Gamma$ is of the form $\gamma=\gamma_{1} \times \gamma_{2} \in \operatorname{Iso}\left(H_{1}\right) \times \operatorname{Iso}\left(H_{2}\right)$, here Iso $\left(H_{i}\right)$ denotes the group of isometries of $H_{i}$ (see Lemma 1 in [3], and the subsequent discussion). Let $p_{i}: \Gamma \rightarrow \operatorname{Iso}\left(H_{i}\right)$ denote the projection homomorphisms, then $\Gamma_{1}=p_{1}(\Gamma)$ acts by translations on $H_{1}$ and $\Gamma_{2}=p_{2}(\Gamma)$ is a discrete subgroup of $\operatorname{Iso}\left(H_{2}\right)$. So $Z \subset \Gamma_{1}$ and $H_{1} / Z$ is a compact flat torus $T^{k}$. The projection $p: H_{1} \rightarrow T^{k}$ allows us to define $\rho: \Gamma_{2} \rightarrow T^{k}$ by setting $\rho\left(p_{2} \gamma\right)=p\left(p_{1} \gamma\right)$. The function $\rho$ is well defined since $\operatorname{ker}(p)=\operatorname{ker}\left(p_{2}\right)=Z$. The centre $Z$ can also be thought of as a set of vectors in $H_{1}$, as $Z \subset \Gamma_{1}$. In this way $Z$ acts on $H_{1}$ by translations.

Recall that $\Gamma_{2}$ has trivial centre and that $M$ is isometric to $\left(T^{k} \times H_{2}\right) / \Gamma_{2}$ [3], here $\Gamma_{2}$ acts on $T^{k} \times H_{2}$ by $\psi(s, h)=(\rho(\psi) s, \psi(h))$ with $(s, h)$ in $T^{k} \times H_{2}$ and $\psi$ in $\Gamma_{2}$. This can be read from the diagram

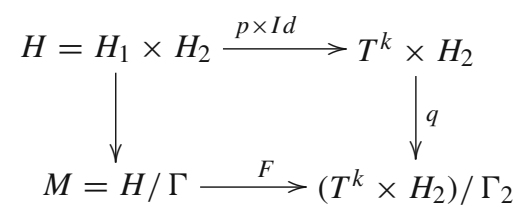

where $F$ is defined so that the diagram commutes.

In $\Gamma_{2}$ there exists a finite index subgroup $\Gamma_{0}$-denoted by $\Gamma_{2}^{* *}$ in [3] - which makes the following diagram commute.

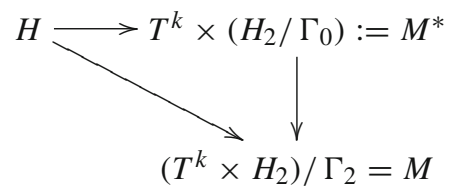

Define $q^{*}: T^{k} \times\left(H_{2} / \Gamma_{0}\right)=M^{*} \rightarrow M$ as in the previous diagram and denote by $\Gamma^{*}$ the group of deck transformations of $q^{*}$ seen as a covering map. Notice that $\Gamma^{*} \subset \Gamma_{2}$.

The function $\rho$ is defined on all of $\Gamma_{2}$, so we can also consider the restriction of $\rho$ to $\Gamma^{*}$. 
We are now in a position to verify that this construction gives $M$ a pure polarised $\mathcal{F}$ structure, and hence $\operatorname{Min} \operatorname{Vol}(M)=0$ as claimed. Let us check that every condition which guarantees the existence of a polarised $\mathcal{F}$-structure is met.

(1) Take $U=M$, as an open cover with a single set.

(2) Define $\tilde{U}:=M^{*}=T^{k} \times N$, here $N=H_{2} / \Gamma_{0}$. The quotient map

$$
q^{*}: \tilde{U}=T^{k} \times N \rightarrow\left(T^{k} \times H_{2}\right) / \Gamma_{2}=M=U
$$

is a finite Galois covering with deck transformation group $\Gamma^{*}$.

(3) The $k$-torus $T^{k}$ acts smoothly and without fixed points on itself as a factor of $T^{k} \times$ $\left(H_{2} / \Gamma_{0}\right)$. So we have the action

$$
\phi: T^{k} \rightarrow \operatorname{Diff}(\tilde{U})=\operatorname{Diff}\left(T^{k} \times N\right)
$$

given by $\phi(t)(s, h)=(s+t, h)$.

(4) We will use the function $\rho$ to define the automorphism $\Psi: \Gamma^{*} \rightarrow \operatorname{Aut}\left(T^{k}\right)$, set $\Psi(\gamma):=$ $\rho(\gamma)$. Let $t \in T^{k}$ and $x \in \tilde{U}=T^{k} \times N \Rightarrow x=(s, h) \in T^{k} \times N$. Notice that for $s$ and $t$ in $T^{k}$ we have that $\rho(\gamma)(s+t)=(\rho(\gamma) s+\rho(\gamma) t)$ since $\rho(\gamma) \in T^{k}$.

We plug in this information to obtain the following equalities:

$$
\begin{aligned}
\gamma(\phi(t) x) & =\gamma(\phi(t)(s, h)) \\
& =\gamma(s+t, h) \\
& =(\rho(\gamma)(s+t), \gamma h) \\
= & (\rho(\gamma) s+\rho(\gamma) t, \gamma h)=\star \\
\phi(\Psi(\gamma) t)(\gamma x) & =\phi(\rho(\gamma) t)(\gamma x) \\
& =\phi(\rho(\gamma) t)(\rho(\gamma) s, \gamma h) \\
& =(\rho(\gamma) s+\rho(\gamma) t, \gamma h)=\star
\end{aligned}
$$

Therefore $\gamma\left(\phi_{i}(t)(x)\right)=\phi_{i}\left(\Psi_{i}(\gamma)(t)\right)(\gamma x)$ and the condition is satisfied.

(5) This condition does not need to be verified, because we only have one covering set.

Since the action of $T^{k}$ on itself as a factor of $T^{k} \times\left(H_{2} / \Gamma_{0}\right)$ is fixed point free, the above construction gives $M$ a pure polarised $\mathcal{F}$-structure.

We will state the contrapositive statement to the theorem because it strengthens Corollary 17 of [5]: Let $M$ be a closed nonpositively curved manifold with positive minimal volume. Then the volume flux group $\Gamma_{\mu}$ is trivial for every volume form $\mu$ on $M$.

Another noteworthy feature of the covering $M^{*} \rightarrow M$ is that on $M^{*} \cong T^{k} \times N$ elements of the volume flux group which come from rotations on the $T^{k}$ factor are circle actions with homologically essential orbits. It is not yet clear if in the general case a non-trivial volume flux group implies existence of a circle action with homologically essential orbits at least in a multiple cover - compare with Remark 19 in [5] — but it is virtually so for every closed nonpositively curved manifold $M$, since it is true for $M^{*}$.

Acknowledgements I wish to warmly thank Dieter Kotschick for a number of interesting conversations and for commenting on a previous version of this work. The author is supported by the Deutsche Forschungsgemeinschaft (DFG) project 'Asymptotic Invariants of Manifolds'. 


\section{References}

1. Bessières, L.: Un théorème de rigidité différentielle. Comment. Math. Helv. 73(3), 443-479 (1998)

2. Cheeger, J., Gromov, M.: Collapsing Riemannian Manifolds while keeping their curvature bounded I. J. Differential Geom. 23, 309-346 (1986)

3. Eberlein, P.: A canonical form for compact nonpositively curved manifolds whose fundamental groups have nontrivial center. Math. Ann. 260(1), 23-29 (1982)

4. Fathi, A.: Structure of the group of homeomorphisms preserving a good measure on a compact manifold. Ann. Sci. École Norm. Sup. (4) 13(1), 45-93 (1980)

5. Kedra, J., Kotschick, D., Morita, S.: Crossed flux homomorphisms and vanishing theorems for flux groups. GAFA 16, 1246-1273 (2006)

6. Kotschick, D.: Entropies, volumes and einstein metrics. Preprint (2004) arXiv : math . DG / 0410215. 